\title{
BMJ Open Substituting physicians with nurse practitioners, physician assistants or nurses in nursing homes: protocol for a realist evaluation case study
}

\author{
Marleen Hermien Lovink, ${ }^{1}$ Anke Persoon, ${ }^{2}$ Anneke JAH van Vught, ${ }^{3}$ \\ Lisette Schoonhoven, ${ }^{1,4}$ Raymond TCM Koopmans, ${ }^{1,5}$ Miranda GH Laurant ${ }^{1,6}$
}

To cite: Lovink MH, Persoon A, van Vught AJAH, et al. Substituting physicians with nurse practitioners, physician assistants or nurses in nursing homes: protocol for a realist evaluation case study. BMJ Open 2017;7:e015134. doi:10.1136/ bmjopen-2016-015134

- Prepublication history for this paper is available online. To view these files please visit the journal online (http://dx.doi org/10.1136/bmjopen-2016015134)

Received 11 November 2016 Revised 13 February 2017 Accepted 13 March 2017

CrossMark

For numbered affiliations see end of article.

Correspondence to

Marleen Hermien Lovink;

marleen.lovink@radboudumc.nl

\section{ABSTRACT}

Introduction In developed countries, substituting physicians with nurse practitioners, physician assistants and nurses (physician substitution) occurs in nursing homes as an answer to the challenges related to the ageing population and the shortage of staff, as well as to guarantee the quality of nursing home care. However, there is great diversity in how physician substitution in nursing homes is modelled and it is unknown how it can best contribute to the quality of healthcare. This study aims to gain insight into how physician substitution is modelled and whether it contributes to perceived quality of healthcare. Second, this study aims to provide insight into the elements of physician substitution that contribute to quality of healthcare.

Methods and analysis This study will use a multiplecase study design that draws upon realist evaluation principles. The realist evaluation is based on four concepts for explaining and understanding interventions: context, mechanism, outcome and context-mechanism-outcome configuration. The following steps will be taken: (1) developing a theory, (2) conducting seven case studies, (3) analysing outcome patterns after each case and a crosscase analysis at the end and (4) revising the initial theory. Ethics and dissemination The research ethics committee of the region Arnhem Nijmegen in the Netherlands concluded that this study does not fall within the scope of the Dutch Medical Research Involving Human Subjects Act (WMO) (registration number 2015/1914). Before the start of the study, the Board of Directors of the nursing home organisations will be informed verbally and by letter and will also be asked for informed consent. In addition, all participants will be informed verbally and by letter and will be asked for informed consent. Findings will be disseminated by publication in a peer-reviewed journal, international and national conferences, national professional associations and policy partners in national government.

\section{INTRODUCTION}

Maintaining the quality of nursing home care in light of the ageing population and the shortages of staff is an important issue in developed countries. Physician substitution is one of the potential solutions used by nursing homes to

\section{Strengths and limitations of this study}

This is the first case study applying a realist evaluation approach in seven cases to gain insight into what mechanism of substituting physicians with nurse practitioners, physician assistants or nurses in nursing homes contributes, in what context and in what respect, to perceived quality of healthcare.

- This case study will build on a theory based on a literature and a focus group study conducted by the research team; the theory is presented in this article.

- All relevant stakeholders involved in physician substitution will be included and different data collection methods will be applied to provide a complete picture of each case which will be input for the cross-case analysis.

- This protocol may guide other researchers in conducting their multiple-case study according to realist evaluation principles.

- Seven cases will be included in this case study; therefore, it might be that not all possible ways of modelling physician substitution in nursing homes are included.

deal with these challenges. ${ }^{1-3}$ However, there is great diversity in how physician substitution in nursing homes is modelled and it is unknown how it can be done best to contribute the most to the quality of healthcare (Lovink et al in preparation).

Physician substitution means shifting care from physicians to nurse practitioners (NPs), physician assistants (PAs) or registered nurses (RNs), also called mid-level providers. We use the term mid-level providers to refer to professionals with European Qualification Level five or higher. ${ }^{4}$ Their introduction in nursing homes has happened for several reasons.

1. The population is ageing, and in this ageing population, the prevalence of (chronic) diseases and multimorbidity is also expected to increase. ${ }^{5}$ 
2. Societal reforms have shifted healthcare from the hospitals and nursing homes to the community. ${ }^{6}$ This means that only patients requiring complex care will reside in nursing homes. As a consequence, attending physicians in nursing homes face heavy workloads. ${ }^{7}$ In the Netherlands, nursing home physician specialists, called elderly care physicians (ECPs), are employed by the nursing home organisation. $^{8} 9$ This is a unique specialty that may contribute to the quality of healthcare. 81011 However, there is also a high workload for ECPs in the Netherlands, and there are many vacancies. ${ }^{12}$

3. Relatively few medical students are pursuing careers in healthcare for older people. ${ }^{12-15}$ By substituting physicians with mid-level providers, these threats to the quality of healthcare may be diminished. ${ }^{2}$

A systematic literature review showed that substituting physicians with mid-level providers in nursing homes appeared to achieve patient outcomes and process of care outcomes that were at least as good as care provided by physicians only. ${ }^{16}$ In addition, a focus group study with care providers of Dutch nursing homes showed that mid-level providers not only substituted for the physicians, but that they had a surplus value, according to the respondents, because they contributed to quality of healthcare, provided patient-centred care and strengthened the care team (Lovink et al in preparation). However, the same study showed that there was great diversity in how physician substitution was modelled and there was no consensus on the optimal way to model physician substitution. Moreover, the results of this focus group study may be distorted by social desirability bias due to self-reporting of activities (Lovink et al in preparation). To gain a more complete and in-depth insight into physician substitution in nursing homes, a multiple-case study will be carried out in seven nursing homes in the Netherlands. This paper describes the study protocol.

\section{STUDY AIM}

The aim of the study is to gain insight into how substitution of ECPs by mid-level providers is modelled and whether it contributes to perceived quality of healthcare. Second, we aim to provide insight into elements of substitution of ECPs by mid-level providers that contribute to quality of healthcare (ie, elements that contribute to an optimal model of physician substitution). In order to do so, the following research questions will be answered:

Research questions:

- How is substitution of ECPs by mid-level providers modelled in different nursing homes?

- What mechanism of substitution of ECPs by midlevel providers contributes, in what context and in what respect, to perceived quality of healthcare for nursing home patients?

-What are the elements that contribute to an optimal model of substitution of ECPs by mid-level providers?

\section{DESIGN}

The study will use a descriptive and partial explanatory multiple-case study design that draws upon realist evaluation principles. ${ }^{1718}$ The realist evaluation is useful for studying complex interventions when the aim of the study is not determining whether an intervention is effective or not, but instead to explain how and why it is effective, under what conditions and for which groups of patients. ${ }^{19}$ The realist evaluation is based on four concepts for explaining and understanding interventions: context $(\mathrm{C})$, mechanism $(\mathrm{M})$, outcome $(\mathrm{O})$ and the contextmechanism-outcome $(\mathrm{CMO})$ configuration. The realist evaluation is a pragmatic alternative to the experimental paradigm, given the impossibility of controlling complex interventions, such as physician substitution. ${ }^{19}$ The following steps will be taken in this study:

1. developing an initial theory (see below);

2. conducting seven case studies (collecting data on (appropriate) contexts, mechanisms and outcomes);

3. analysing outcome patterns after each case and a cross-case analysis at the end to see which can and which cannot be explained by the initial theory;

4. revising understanding of $\mathrm{CMO}$ configurations as a prelude to a further theory refinement.

\section{INITIAL THEORY SUBSTITUTION}

In the following paragraphs, a theory of substitution of ECPs by mid-level providers in nursing homes will be presented according to the concepts of the realist evaluation. ${ }^{18}$ Realist evaluation starts with eliciting and formalising the theory to be tested. In addition, data will be collected and analysed, and the theory will be tested. ${ }^{19}$ The initial theory presented is partly based on literature and partly on a focus group study we performed (Lovink et al in preparation). In the focus group study, ECPs, NPs, PAs and RNs (in total, 35 care providers) working in Dutch nursing homes were interviewed about the topic of physician substitution. The theory is a preliminary theory that will be adjusted and further developed in this case study. Below, it is presented under the headings Mechanisms, Contexts and Outcomes, starting with the heading Mechanisms, as this is the core of CMO configurations. This theory (depicted in figure 1) will be the starting point for the case study. If no reference is provided, the information is based on our focus group study (Lovink $e t$ $a l$ in preparation).

\section{Mechanisms}

Mechanism describes what it is about the intervention that brings about any effect. ${ }^{19}$ Below are presented three head mechanisms. Figure 1 presents the underlying mechanisms.

\section{Mechanism 1}

Based on their education and previous experience, mid-level providers are able to substitute for ECPs largely 


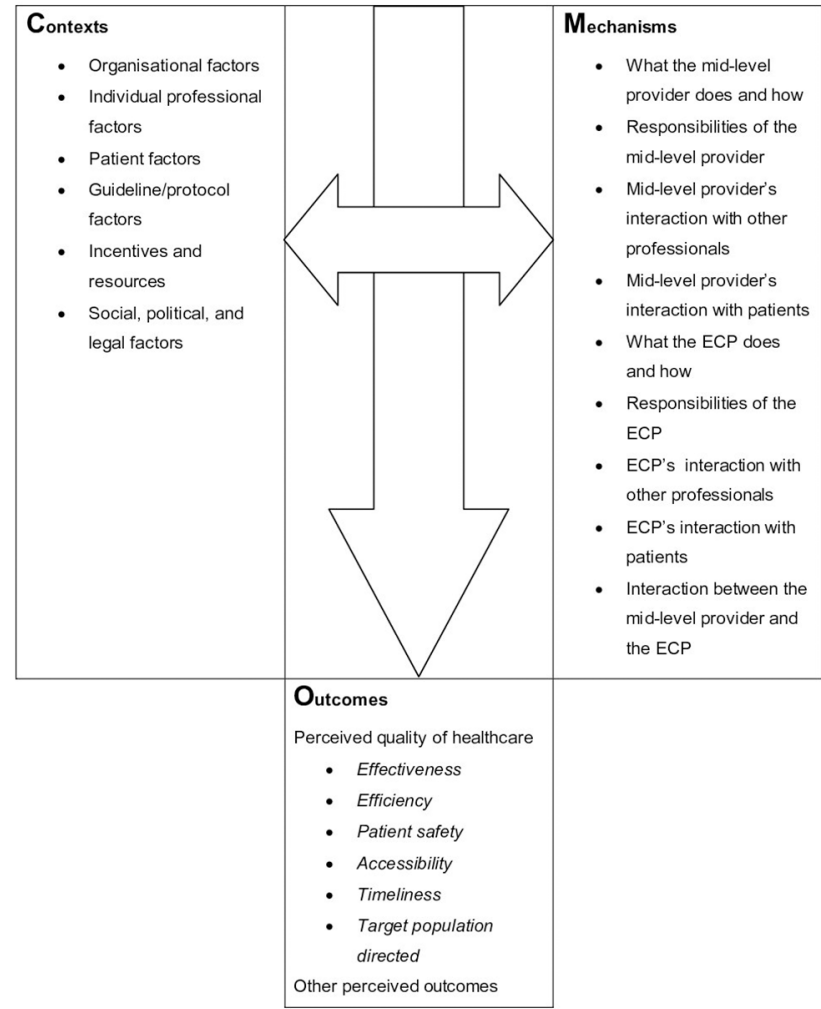

Figure 1 Interpretive framework of substitution of elderly care physicians (ECPs) by mid-level providers

autonomously with at least maintenance of the quality of healthcare.

In the Netherlands, NPs were introduced in the late $1990 \mathrm{~s} .{ }^{20} \mathrm{NPs}$ are RNs with completed advanced education and clinical training on a master's level. They can provide a wide range of preventive, chronic healthcare and acute healthcare in a wide variety of clinical areas. While NPs combine nursing care with medical care, PAs mainly provide medical care. ${ }^{21}$ PAs were introduced in the Netherlands in the early 2000s. ${ }^{22}$ The PA course is a graduate programme that leads to a master's degree and the programme consists of a didactic phase and a clinical phase. ${ }^{22}$ PAs work across a wide range of healthcare settings and in a wide variety of clinical areas. Following the example of general practices, more and more practice nurses started working in nursing homes in the Netherlands the last decades. ${ }^{23}$ Practice nurses in nursing homes are nurses with additional training on older patients and the nurse's role in nursing homes. NPs, PAs and practice nurses all have the potential to reduce ECPs' workload and to contribute to the quality of healthcare in the unique multidisciplinary nursing home setting in the Netherlands. ${ }^{24} 25$

NPs and PAs are able to substitute for ECPs. ${ }^{26-29}$ PAs mostly substitute for ECPs to a large extent with regard to medical tasks, while the extent to which NPs can substitute for ECPs varies from a smaller to a larger extent. In addition, the level of autonomy of the NP/PA in the medical domain varies from one nursing home to another. ${ }^{30} \mathrm{PAs}$ mostly have a high level of autonomy; they perform most of their tasks independent of an ECP. The level of autonomy of NPs varies. NPs/PAs can work at all different units of a nursing home: units for patients with physical disabilities, dementia special care units, or geriatric rehabilitation units, or a combination of different units. Tasks that can be replaced from ECPs to NPs or PAs are admission of patients, assessment and management and follow-up of patients with a variety of chronic conditions, as well as acute conditions, determining patients' care plan, visits, multidisciplinary meetings, family meetings, procedures such as prescription of medication, referral to other disciplines, out-of-hours care, and so on. ${ }^{25} 2628$ Furthermore, some NPs/PAs in nursing homes work as a specialist at the organisational level. ${ }^{21}{ }^{25}$ Some PAs work as a specialist in addition to their work as a generalist, while some NPs only work as a specialist. Examples of specialist areas are wound care, pressure ulcers and diabetes mellitus.

Practice nurses can also substitute for ECPs. ${ }^{25}$ The extent to which they can be a substitute on medical tasks and their level of autonomy in the medical domain is mostly lower than is the level of autonomy of NPs/PAs. Practice nurses mostly work at units for patients with physical disabilities or dementia special care units. They may work at one or more units in the organisation and they may work as a specialist at the organisational level. ${ }^{25}$ Tasks they can perform are visits (in preparation for the ECP's visit), triage, wound rounds, and so on. ${ }^{25}$

Although the above indicates that physician substitution in nursing homes is possible, it also indicates that there is great diversity in how it is modelled and the elements of an optimal model are unknown.

\section{Mechanism 2}

Physician substitution always is a collaboration between the mid-level provider and the ECP to guarantee quality of healthcare. The role of the ECP changes due to this collaboration.

The level of collaboration between mid-level providers and the ECP varies. In some cases, the NP/PA has structural meetings with the ECP, while in other cases, the NP/PA only consults with the ECP if needed. ${ }^{25}{ }^{28}$ Practice nurses perform most of their tasks under the supervision of ECPs. ${ }^{25}$ Trust and a 'personal click' seem to be important factors for a successful collaboration.

By shifting care to mid-level providers, the ECPs can spend more time on complex care or special areas of attention, such as palliative care. For less complex care, the role of the ECPs will become more of a coordinating role due to substitution of ECPs by mid-level providers. Furthermore, the ECPs are able to provide care to older adults living at home as a consultant for the general practitioner. Although physician substitution releases the burden on ECPs during the day, the burden during evening, night and weekend shifts may increase because, in most cases, mid-level providers are employed instead of an ECP but they do not participate in these off-hours shifts (resulting in the same number of shifts with fewer people). 


\section{Mechanism 3}

Mid-level providers have a different way of working and they perform additional tasks compared with ECPs, which may lead to an increased quality of healthcare.

During the performance of their tasks, mid-level providers show, to a more or lesser extent, the following characteristics: closeness to the patient/family, strengthening of the care team and acting as a bridge between the ECP and the care team and the patient or the family. ${ }^{21252830}$ NPs and practice nurses show these characteristics more than do PAs. ${ }^{20}$ In addition to the patient-related tasks, mid-level providers perform non-patient-related tasks as well, such as teaching and coaching of the care team, innovation of healthcare and innovation of the organisation of healthcare. ${ }^{25-30}$

\section{Contexts}

The context are those features of the conditions that are relevant to the operation of the mechanism. ${ }^{19}$

The factors that influence the level of physician substitution and the role of the mid-level provider in nursing homes can be classified according to the seven domains of the 'Tailored Implementation for Chronic Diseases' checklist: (1) organisational factors; (2) individual professional factors; (3) patient factors; (4) guideline factors; (5) incentives and resources; and (6) social, political and legal factors. ${ }^{16} 3031$ The seventh domain, "professional interactions', is seen as part of the mechanism.

\section{Organisational factors}

Organisational factors that influence the level of physician substitution are the demographics of an organisation (eg, number of patients), the vision of the organisation on physician substitution and how the mid-level provider is positioned in the organisation. For example, in an organisation with a shortage of ECPs, the role of the mid-level provider (to substitute the ECP) will be mainly focused on care delivery, which might be different than in an organisation without a shortage of ECPs, where the role of the mid-level provider may be more focused on quality improvement. In addition, whether or not mid-level providers and ECPs form fixed couples or rotate influences the consistency of care and the level of trust in one another. ${ }^{16}$ Furthermore, some nursing homes introduce the mid-level provider in their organisation without a clear vision of their role; this may hinder the implementation of physician substitution as the role of the mid-level provider is not clear to ECPs. When mid-level providers are positioned in the nursing team, their role will be different from cases in which mid-level providers are positioned in the medical team next to the ECP, which facilitates physician substitution. Another important factor is that the position of the mid-level provider needs time to embed in a nursing home organisation.

\section{Individual professional factors}

Individual professional factors influence the role of the mid-level provider, especially the characteristics of the mid-level provider himself/herself, of the ECP, and of the care team and other care providers. Characteristics of the mid-level provider himself/herself are, for example, type of mid-level provider (NP, PA or practice nurse: see 'Mechanisms' section), background and level of experience. A pioneering spirit, ability to work independently, thirst for knowledge and willingness to shape his or her own practice contribute to successful implementation of the mid-level provider position. ${ }^{16}$ In addition, the willingness of the ECP to substitute tasks shapes the role of the mid-level provider. ${ }^{16}$ An example of a characteristic of the care team, which influences the role of mid-level providers, is the level of education. If this level is low, the mid-level provider will be inclined to work in the nursing domain instead of the medical domain. The level at which other care providers accept the mid-level provider also influences their role and the ease of performing their role.

\section{Patient factors}

Characteristics of the patients that influence the role of mid-level provider are, for example, their type of care needs. In the Netherlands, there is a difference between units for patients with physical disabilities and dementia special care units, and geriatric rehabilitation units are often part of a nursing home. ${ }^{24}$ Mid-level providers may work at all units; however, the type of unit determines their tasks. Another characteristic of patients can include their familiarity with the function of mid-level providers; if they are not familiar, they might demand to be taken care of by an ECP. ${ }^{16}$

\section{Guideline factors}

Substitution of ECPs by mid-level providers is strongly influenced by the agreements, or lack thereof, made regarding substitution. Examples of agreements are vision on physician substitution, job description of the mid-level provider, collaborative agreements and treatment protocols that are adjusted to the mid-level provider based on the scope of practice.

\section{Incentives and resources}

Appropriate financing is an important factor for successful implementation of the mid-level provider in nursing homes. ${ }^{16}$ This includes financing at the organisational level-how the employment of a mid-level provider is reimbursed-and at professional level, remuneration that is appropriate for the task and responsibilities of the mid-level provider.

\section{Social, political and legal factors}

The support of the mid-level provider as an ECP substitute from the professional association of ECPs is an important factor related to the acceptation of mid-level providers. Political and legal factors are also context features of physician substitution. These factors determine the boundaries of mid-level providers' authorisation and they determine when, how, where and by whom healthcare for older people is provided. ${ }^{16}$ In the Netherlands, NPs 
and PAs are authorised to indicate and perform some of the so-called 'reserved procedures' described in the Individual Health Care Professions Act, which were initially only reserved for physicians. Furthermore, NPs and PAs are not authorised to sign death certificates. ${ }^{32} 33$ Practice nurses are only allowed to perform reserved procedures after instructions from a physician, NP or PA. In addition, the ageing population and the societal reforms that shift care from the hospital/nursing home to the community influence the way mid-level providers are employed.

\section{Outcomes}

Outcome patterns are comprised of the intended and unintended consequences of the intervention. ${ }^{19}$

The outcomes of physician substitution will be discussed as outcomes related to quality of healthcare based on the six concepts of quality of healthcare defined by the Ministry of Health, Welfare and Sport of the Netherlands (2014) and WHO (2006): (1) effectiveness, (2) efficiency, (3) patient safety, (4) accessibility, (5) timeliness and (6) target population directed. ${ }^{3435}$

\section{Effectiveness}

Effectiveness refers to delivering healthcare that is adherent to an evidence base and results in improved health outcomes for individuals and communities, based on needs. ${ }^{34} 35$

Substitution of ECPs by NPs or PAs seems to have a neutral effect on or cause a reduction in the number of hospital admissions, hospital days, emergency department visits, mortality and number of medications used. ${ }^{16}$ The effectiveness of substitution of ECPs by practice nurses is unknown.

\section{Efficiency}

\section{Efficiency refers to healthcare that avoids waste. ${ }^{34} 35$}

Physician substitution appears to have a mixed effect on healthcare use (costs). ${ }^{16}$ However, if mid-level providers perform the same activities as an ECP, they do this at lower costs because of their lower salary. In contrast to this, mid-level providers hardly ever fully replace ECPs. ${ }^{25}$ The NP may supply a time savings for the ECP of between $40 \%$ and $88 \%$ and the practice nurse, between $35 \%$ and $72 \% .{ }^{25}$ The time savings a PA supplies is unknown. It is unknown how the lower costs (salary) of mid-level providers relate to the substitution percentage in terms of efficiency. In addition, mid-level providers contribute to efficiency as they work in a structured manner and take into account the organisation of care while planning care activities.

\section{Patient safety}

Patient safety refers to avoiding harm during healthcare interventions. ${ }^{34} 35$

Mid-level providers seem to be able to substitute for ECPs in terms of maintaining patient safety within their boundaries and if an ECP is available for support if needed. In addition, mid-level providers might detect medical problems early because they are regularly present on the units. They might also focus on the quality policy, such as developing protocols and stimulating working according to these protocols. ${ }^{25}$

\section{Accessibility}

Accessibility refers to how easily someone obtains access to healthcare, which does not vary in quality because of personal characteristics such as gender, race, ethnicity, geographical location or socioeconomic status. ${ }^{34} 35$

Mid-level providers may enhance the accessibility of medical care. They are easily accessible to the care team as well as for patients and family because they are often present at the unit and have an open attitude. ${ }^{25}$

\section{Timeliness}

Timeliness refers to providing healthcare in time..$^{34} 35$

NPs appear to provide as many progress visits as ECPS, while NPs perform more acute visits. ${ }^{16}$ In addition, mid-level providers may have/take more time for direct patient care than do ECPs.

\section{Target population directed}

Target population directed refers to respecting the preferences, needs and values of the target group. ${ }^{34} 35$

Mid-level providers may contribute to target population directness because they know their patients very well, involve family in decisions and communicate with patients and family on their own level.

\section{Other outcomes ('indirect outcomes'19)}

Mid-level providers may contribute to the continuity of care as they work at one place for a long time. ${ }^{25}$ In addition, the fact that mid-level providers perform different tasks and have a different way of working than ECPs may lead to better quality of healthcare, but also to other outcomes. For example, coaching of the care team during a training may lead to increased knowledge of the care team. As the goal of this study is to describe physician substitution, we did not focus explicitly on 'indirect' outcomes, but they might be discussed in answers to our open interview questions and then will be included in the analysis.

\section{METHODS}

\section{Case selection}

The goal of a case study is not statistical generalisation, but analytic generalisation. This means that the initially developed theory is used as a template with which the empirical results of the case study are compared. Each case must be adequately selected so that it either (1) predicts similar results (literal replication) or (2) predicts contrasting results for anticipated reasons (theoretical replication) ${ }^{17}$ In this study, each case will be comprised of one mid-level provider in a nursing home organisation. The first mechanism: mid-level providers can substitute for ECPs largely autonomously, at least in terms of maintenance of quality of healthcare. This is the mechanism we are most interested in and therefore, this mechanism will guide the case selection. The main goal of the 


\section{Box 1 Selection criteria}

\section{Inclusion criteria}

$>>65 \%$ of the patient-related tasks ${ }^{*}$ the mid-level provider performs should be in the medical domaint, according to the mid-level provider's own estimation.

- The mid-level provider should be employed for minimal 0.6 full time equivalents.

$-\geq 80 \%$ of the patients the mid-level provider takes care of should be 65 years or older.

- If possible (depending on the available cases), the mid-level provider should be working for $>2$ years as a mid-level provider in a nursing home.

\section{Maximum variation criteria}

- Level of autonomy $\neq(>70 \% /<70 \%)$, in the performance of patientrelated tasks in the medical domain, according to the mid-level provider's own estimation.

- Working as a generalist, or a specialist, or both.

- Working at unit level, or at organisation level, or both.

- Working at unit for patients with physical disabilities, dementia special care unit, or geriatric rehabilitation unit, or a combination of different units.

- Type of mid-level provider (nurse practitioner/physician assistant/ practice nurse).

Male, female.

*Patient-related tasks: direct patient-related tasks and indirect patient-related tasks. Direct patient-related tasks: tasks that are performed in the presence of/with the patient and/or family. Indirect patient-related tasks: tasks that are performed for the patient, but not per se in the presence of the patient. †Medical domain: medical examination of the patient (history, physical examination, etc), medical diagnostics, formulate a medical treatment plan, indicate and/or perform medical procedures (prescription of medication, perform surgical procedures, give injections, etc).

¥Autonomy: independent indication and performance of patient-related tasks in the medical domain. The performance can also be delegated to another care provider. Consultation with an elderly care physician is possible, but the mid-level provider is responsible.

selection is to select cases in which the mid-level provider works mainly in the medical domain. To gain insight into whether or not mid-level providers can substitute for ECPs largely autonomously, at least in terms of the maintenance of quality of healthcare, we will seek variation on the level of autonomy. We will also seek variation on other factors of the first mechanism. See box 1 for a description of the selection criteria.

The professional associations of NPs, PAs and practice nurses in nursing homes will be asked to distribute a questionnaire among their members (NPs: 224; PAs: 30; practice nurses: 180). This questionnaire contains questions about the inclusion criteria and the maximum variation criteria. Reminders will be used to enhance the response rate. The completed questionnaires will be used to select seven cases. The number of seven was chosen to create a balance between depth and variation in the study with the given budget and time available.

\section{Setting}

The setting will be seven nursing home organisations in the Netherlands that have one or more locations, and the (different) unit(s) where the mid-level providers work.

\section{Participants}

The participants will be

- the mid-level provider;

- the manager that has been/is involved the most in the decision to substitute for ECPs;

- the supervisor/manager of the mid-level provider;

- the head ECP;

- all ECPs with whom the mid-level provider collaborates directly;

- five nurses/healthcare assistants/nursing team leaders with whom the mid-level provider collaborates;

- five patients the mid-level provider takes care of and their informal caregiver (at dementia special care units; only informal caregivers will participate);

- patient council, family council or patient-family council.

\section{Data collection}

Before the start of the study, the Board of Directors of the nursing home organisations will be informed verbally and by letter and they will be asked to provide informed consent for the entire study. In each case, two researchers (MLo and IM) will collect all data in 2 weeks. Data collection will consist of observations, interviews, questionnaires and documents (see table 1). All interviews will be audiotaped and transcribed verbatim. Data will be collected between September 2015 and January 2017.

\section{Informed consent}

All participants who will be interviewed will be informed verbally and by letter and will be asked to provide informed written consent. A contact person (eg, manager, nursing team leader) and/or the mid-level provider will assist in identifying all participants. The contact person will draw a random sample of five nurses/healthcare assistants/ nursing team leaders. With the help of the contact person and/or the mid-level provider, patients will be selected for an interview. Five patients who are 65 years or older and mentally competent (according to the judgement of the contact person or the mid-level provider) will be asked for an interview, together with his/her informal caregiver. On dementia special care units, only the informal caregiver will be interviewed. In addition, the patient/family council will be contacted via the mid-level provider and the members will be invited for a focus group interview, as well as to sign an informed consent.

Before the start of the study, all patients, informal caregivers and care providers of the units where observations will take place will be informed about the study and the observations, so they have the chance to object to the observation in advance. The method for informing participants about the observations will be determined in 


\section{Table 1 Data collection}

\begin{tabular}{|c|c|}
\hline Sources of data & Data \\
\hline $\begin{array}{l}\text { Mid-level provider } \\
\text { (three NPs, two PAs } \\
\text { and two practice } \\
\text { nurses) }\end{array}$ & $\begin{array}{l}\text { Observation ( } 4 \times 4 \text { hours) } \\
\text { Questionnaire } \\
\text { Interview (after observation) }\end{array}$ \\
\hline $\begin{array}{l}\text { Manager involved in } \\
\text { physician substitution }\end{array}$ & $\begin{array}{l}\text { Questionnaire } \\
\text { Interview }\end{array}$ \\
\hline $\begin{array}{l}\text { Supervisor/manager of } \\
\text { the mid-level provider }\end{array}$ & Interview \\
\hline $\begin{array}{l}\text { ECPs with whom the } \\
\text { mid-level provider } \\
\text { collaborates most } \\
\text { intensely }\end{array}$ & $\begin{array}{l}\text { Observation ( } 2 \times 2 \text { hours) } \\
\text { Questionnaire } \\
\text { Interview (after observation) }\end{array}$ \\
\hline $\begin{array}{l}\text { ECPs with whom the } \\
\text { mid-level provider } \\
\text { collaborates directly }\end{array}$ & $\begin{array}{l}\text { Questionnaire } \\
\text { Interview }\end{array}$ \\
\hline Head of the ECPs & - Interview \\
\hline $\begin{array}{l}\text { Five nurses/healthcare } \\
\text { assistants/nursing team } \\
\text { leaders with whom } \\
\text { the mid-level provider } \\
\text { collaborates }\end{array}$ & Interview \\
\hline $\begin{array}{l}\text { Five patients the mid- } \\
\text { level provider takes } \\
\text { care of and/or their } \\
\text { informal caregiver }\end{array}$ & Interview \\
\hline $\begin{array}{l}\text { Patient council, family } \\
\text { council or patient- } \\
\text { family council }\end{array}$ & Focus group interview \\
\hline Documents & $\begin{array}{l}\text { Mission and vision of the } \\
\text { organisation } \\
\text { Mission and vision of the } \\
\text { organisation on physician } \\
\text { substitution } \\
\text { Job description of all mid-level } \\
\text { providers in the organisation and } \\
\text { of the ECP } \\
\text { Working arrangements for the } \\
\text { mid-level provider and the ECP } \\
\text { Treatment protocols for the mid- } \\
\text { level provider } \\
\text { Annual report of the organisation } \\
\text { of the preceding year } \\
\text { Information about the mid-level } \\
\text { provider for patients and family }\end{array}$ \\
\hline
\end{tabular}

ECPs, elderly care physicians; NPs, nurse practitioners; PAs, physician assistants.

collaboration with our contact person and the Board of Directors.

During the observations, all patients that the mid-level provider and the ECP visit will receive brief information about the study and then will be verbally asked for informed consent to observe the contact with the mid-level provider or ECP (ie, a written informed consent form will not be used). This will be the same for all care providers that the mid-level provider/ECP has contact with during the observations.

\section{Observations}

Observational guides are developed based on the framework depicted in figure 1 . The mid-level provider will be observed for 4 days $\times 4$ hours within the 2 -week period and the ECP for 2 days $\times 2$ hours within the 2 -week period. These time periods have been chosen as it is anticipated that an observation of 2 or 4 hours gives a good impression of the tasks the mid-level provider and the ECP perform. By planning multiple observations, the chance of only observing exceptional situations is diminished. The mid-level provider will be observed for a longer period of time as he/she is the subject of the study. In addition, within the observation of the mid-level provider all scheduled contact moments between the mid-level provider and the ECP will be observed. The ECP will be observed to discover differences or similarities in performing the tasks they have in common with the mid-level provider. Observations will be planned in advance based on indication of the mid-level provider and the ECP which time they perform the most patient-related tasks. Both researchers will carry out half of the observations. The role of the researcher during observations will be as a non-participant. ${ }^{36}$ In non-participant observation, it is important to find a balance between building trust among the participants and 'going native'. The relatively short observation periods will prevent the observers 'going native'. The observational instrument consists of two parts. In one part, the researcher will write down what tasks the mid-level provider performs and how he/she performs these tasks. In the second part, the researcher will write down a general impression on topics such as level of autonomy and care for the client/family after each observation moment. The field notes in the first part of the observation instrument can be used to fill out the second part. After each observation moment, the researcher will directly type out the field notes on a computer.

\section{Interviews and questionnaires}

The interview guides will be developed based on the framework depicted in figure 1, with a different focus for each group of participants. The interview with the mid-level provider will be very extensive and will focus on all relevant items; the interview with the manager will mainly focus on the vision of the organisation on physician substitution and the interview with patients and/or their informal caregiver will mainly focus on their needs and their experiences with the mid-level provider. Tasks and responsibilities will be collected via a questionnaire for the mid-level provider and the ECPs with whom the mid-level provider collaborates directly. The specific outcomes (see figure 1) will be inquired about in the interviews with the mid-level provider, the ECPs with whom the mid-level providers collaborate directly and the nurses/ healthcare assistants/nursing team leaders with whom 
the mid-level provider collaborates. Participants will be asked to compare the mid-level provider and the ECP on all of these outcomes. In the other interviews, outcomes will be discussed in general. In addition, all participants, except for the patients and/or their informal caregiver and the patient/family council, will be asked whether they perceive the way physician substitution is modelled as being optimal and why they think so or not. They will also be asked whether they would recommend it to other organisations and why they would or would not. After analysis of each case, a member check (confirmatory focus group interview) will be carried out. See 'Data analysis' section for further details.

\section{DATA ANALYSIS}

Data will be analysed in the 5 weeks directly after data collection of each case. At completion of the initial analysis of all cases at the end of the study, a cross-case analysis will be carried out.

The data analysis will rely on theoretical propositions and explanation building. This means that the theoretical propositions (the initial theory) that led to this case study will be followed and that the analysis aims to answer the questions: (1) How is substitution of ECPs by mid-level providers modelled in different nursing homes? (2) What mechanism of substitution of ECPs by mid-level providers contributes, in what context and in what respect, to perceived quality of healthcare for nursing home patients? (3) What are the elements that contribute to an optimal model of substitution of ECPs by mid-level providers?

\section{Single-case analysis}

\section{Qualitative analysis}

The tasks in the first part of the observation instrument will be coded according to the possible tasks described in advance. However, there is also space for tasks that are not described in advance. Each observation moment will be coded by one researcher and checked by the other.

The two researchers who collect the data (MLo and IM) will compare their notes in the second part of the observation instrument-the general impression. Differences will be discussed, and finally, they will make an assembly of the different forms. If no consensus can be reached, they will ask clarification during the member check (see below).

Four researchers (MLo, IM, AvV and LvD) will qualitatively analyse the interviews and documents. MLo will code all interviews. In the first case, a second researcher will independently code all interviews. If sufficient consensus is reached in the coding, for the next cases, half of the interviews will be coded independently by a second researcher; for the other half, MLo's codes will be checked by another researcher. The computer program ATLAS.ti will be used for analysis. Content analysis will be used to analyse the data. ${ }^{37}$ This is a method to attain both condensed and broad descriptions of a phenomenon by analysing text data. ${ }^{37}$ The developed theory of context, mechanism and outcome will be tested using deductive coding. This means that a structured categorisation matrix based on figure 1 will be used. However, aspects that do not fit the categorisation matrix will be used to create new categories based on the principle of inductive content analysis. $^{37}$

The researchers who collect the data will use the method of 'outlining the main message'. ${ }^{38}$ The researchers will pretend that the deadline to hand in the final case description is imminent and they will ask themselves the question: how would the main message of this case be formulated? ${ }^{38}$ This question focuses the researcher to think about the content of the result section. Both researchers will do this independently during analysis and they will compare and discuss their main message. In addition, they will check their main message with the data collected.

\section{Quantitative analysis}

The questionnaires and the quantitative parts of the interviews (demographic data) will be quantitatively analysed. The computer program SPSS Statistics V.20 will be used for analysis. Data will be analysed using descriptive statistics.

\section{Member check}

For each individual case, MLo will write a case description and the other researchers will check it. This description will build on the theoretical propositions made at the start of this case study. This description will be used for a member check within the case. ${ }^{3839}$ The mid-level provider, the ECP that has been observed, the manager involved in physician substitution, the manager/supervisor of the mid-level provider and two members of the care team will be asked to read the case description. In a focus group, these participants will be asked whether the case description is an accurate description of their case and clarification on the parts that turned out to be unclear will be asked. The member check has some drawbacks, such as participants struggling with abstract synthesis, participants that want to change their initial response and participants with different views on the same data. ${ }^{40}$ To face these drawbacks, a focus group will be organised so that the interaction process can provide additional information, helping to make it clear why someone struggles with abstract synthesis, why someone has changed his or her mind or why participants have different views. All of this information will enrich the case description. The information gathered during the focus group will be used to further develop the case description.

\section{Cross-case analysis}

When the initial analysis of each case is completed, the process of realistic cumulation will begin. ${ }^{18}$ This means a motion up and down the ladder of abstraction and specification; the data gathered will be used to further develop the 'abstract' theory of physician substitution in 
nursing homes. The cross-case analysis will go beyond the separate Cs, Ms and Os. For each case, the CMO configurations will be determined based on the initial analysis by answering questions like which elements of the mechanism and the context give what outcomes. These CMO configurations will be developed at case level. Where outcomes are unknown, anticipated outcomes (in line with the collected data) will be formulated. In addition, CMO configurations across cases will be determined. ${ }^{41} 42$ At the end, these CMO configurations will help us answer the research questions.

\section{VALIDITY AND RIGOUR}

The trustworthiness of the study findings is based on the following four criteria: (1) credibility, (2) dependability, (3) confirmation and (4) transferability. ${ }^{39}$

- Credibility will be ensured by the selection of seven different cases according to inclusion criteria and maximum variation sampling. In addition, all relevant stakeholders involved in physician substitution will be included and a member check will be performed in each case. The collection of different types of data, known as data triangulation, also contributes to the credibility. To diminish the observer effect, ${ }^{36}$ the researchers will explain to the care provider being observed that there is no good or bad behaviour and that the goal of the observation is only to describe the case and not to judge the behaviour.

- Dependability will be promoted by thoroughly analysing and involving all researchers in the crosscase analysis.

- Confirmation will be enhanced by keeping a logbook on methodological issues, in addition to memos reflecting on their role during the observations and interviews. Both researchers are health scientists with a nursing background. They are aware of the fact that their background may cause them to focus more on the nursing domain than on the medical domain during data collection and analysis.

During non-participant observations, it is a challenge to remain objective and not selective. ${ }^{36}$ Dealing with this challenge starts with acknowledging that an observer can never be truly objective and will always be somewhat selective. ${ }^{43}$ Objectivity will be enhanced through the collection of field notes from two researchers, observations during different moments, structured data collection, check of the observers' ideas on the main message relative to the collected data, discussions of the findings in the research team and the member check.

Prior to the start of the case study and the research proposal, the observation instruments were tested by the two researchers (MLo and IM) using an ECP and a NP, both for 4 hours. After the observations, they discussed and compared their field notes and discussed their role during observations. After this test, they made changes to the observation instru- ments, in addition to making decisions on the focus during observations (the mechanism) and on their role during observation (eg, introduce oneself with a handshake). By performing the test, the researchers developed the observation instrument, as well as establishing themselves as a data collection instrument.

- Transferability: a general description of the organisations that provides sufficient information to implement a similar role and model of care will be presented in the paper to be published.

\section{DISCUSSION}

This case study will provide insight into how substitution of ECPs by mid-level providers is modelled in different nursing homes and what mechanism contributes in what context and in what respect to quality of healthcare for older people. In addition, it will give input for the most optimal model of physician substitution in nursing homes. As stated in the preliminary theory, the model might strongly depend on the context, so there might be no single best model. Furthermore, each model studied in this case study might have strong and weak parts. Therefore, the most optimal model (for a given context) might consist of a combination of parts of different models. Bryant and DiCenco developed the PEPPA framework: participatory, evidence-based, patient-focused process for advanced practice nursing role development, implementation, and evaluation. This framework states that the role of an advanced practice nurses should be developed based on a needs assessment and clear goals, objectives and outcomes identified. ${ }^{44}$ A model might be optimal if the role of a mid-level provider is developed in this manner. In addition to this framework, this case study will provide some concrete examples of this general statement and concrete preconditions of implementing a mid-level provider.

This study is conducted in the Netherlands and it is important to point out that the nursing home setting might differ from other countries. In the Netherlands, multidisciplinary teams are employed by the nursing home organisations, including the ECP, physiotherapist, occupational therapist, speech therapist, dietician and psychologist. ${ }^{8945}$ This means that all these providers are full time present at the nursing home and not only on call. Worldwide the employment of a broad multidisciplinary team is unique, especially the presence of an ECP as a medical specialist in elderly care. ${ }^{75}$ The cooperation between the Dutch ECPs and the relatively new mid-level providers will be influenced positively as well as negatively, as it is facilitated by the presence of the ECPs, but possibly hindered by competition. The interaction between the ECP and the mid-level provider and how this interaction influences physician substitution is part of the current study in observations as well as in interviews, resulting in recommendations on how to strengthen the cooperation.

Besides the differences in the nursing home setting, there is also a huge difference in the extent of substitution 
of physicians by NPs and PAs between countries. As in other countries, PAs in the Netherlands mainly focus on the medical domain, while NPs combine the medical with the nursing domain. In the Netherlands, NPs and PAs are educated at the master's level, they have a protected title and are authorised to indicate and perform some of the so-called 'reserved procedures', like prescribing medication and giving injections. ${ }^{32} 33{ }^{46}$ Research shows that in some countries (like Australia and the USA) NPs are able to substitute physicians like in the Netherlands, while in other countries (like France and Germany) they are not. ${ }^{47}$ For PAs applies that like in the Netherlands they are also recognised in Australia, Canada, the UK and the USA, but in these countries they are only allowed to work under a supervising physician. ${ }^{48}$

This case study will build on a theory based on the literature and a focus group study conducted by the research team. The challenge of performing a case study with certain propositions is to keep an open mind while collecting data. ${ }^{17}$ Although the theory will guide data collection and analysis, it must not confine the data collection and analysis process; there has to be room for alternative hypotheses. The research team will face this challenge by being aware of a vision that is too narrow during data collection and discussing the theory and alternative hypotheses in regular meetings. In this case study, all outcomes are perceived outcomes and no quantitative outcomes are measured. This should be taken into account while interpreting the results. It might be that we cannot 'complete' some CMO configurations because the outcome of a certain mechanism in a certain context is not fully clear. However, this case study will provide insight into the possible outcomes related to physician substitution in nursing homes, which might inform further research.

The results of this case study will inform care providers, managers and policy administrators in their decisions regarding how to substitute mid-level providers for ECPs in nursing homes in a way that contributes most to perceived quality of healthcare for older people.

\section{Author affiliations}

${ }^{1}$ Radboud University Medical Center, Radboud Institute for Health Sciences,

Scientific Center for Quality of Healthcare (IQ Healthcare), Nijmegen, the

Netherlands

${ }^{2}$ Department of Primary and Community Care, Radboud university Medical Center, Radboud Institute for Health Sciences, the Netherlands

${ }^{3}$ HAN University of Applied Sciences, Faculty of Health and Social Studies, Nijmegen, the Netherlands

${ }^{4}$ University of Southampton, Faculty of Health Sciences, Southampton, UK ${ }^{5}$ Joachim \& Anna, Center for Specialized Geriatric Care, Nijmegen, the Netherlands ${ }^{6}$ HAN University of Applied Sciences, Faculty of Health and Social Studies, Nijmegen, the Netherlands

Contributors AP, RK and MLo conceived the idea for the study and obtained funding. All authors have been involved in designing the study. MLo drafted the manuscript for submission to BMJ Open. All authors have been involved in revising the manuscript. All authors read and approved the final manuscript.

Competing interests None declared.

Ethics approval The research ethics committee of the region Arnhem Nijmegen in the Netherlands.
Provenance and peer review Not commissioned; externally peer reviewed.

Open Access This is an Open Access article distributed in accordance with the Creative Commons Attribution Non Commercial (CC BY-NC 4.0) license, which permits others to distribute, remix, adapt, build upon this work non-commercially, and license their derivative works on different terms, provided the original work is properly cited and the use is non-commercial. See: http://creativecommons.org/ licenses/by-nc/4.0/

(C) Article author(s) (or their employer(s) unless otherwise stated in the text of the article) 2017. All rights reserved. No commercial use is permitted unless otherwise expressly granted.

\section{REFERENCES}

1. Donald F, Martin-Misener R, Carter N, et al. A systematic review of the effectiveness of advanced practice nurses in long-term care. $J$ Adv Nurs 2013;69:2148-61.

2. Caprio TV. Physician practice in the nursing home: collaboration with nurse practitioners and physician assistants. Ann Longterm Care 2006;14:17-24.

3. Intrator O, Miller EA, Gadbois E, et al. Trends in nurse practitioner and physician assistant practice in nursing homes, 2000-2010. Health Serv Res 2015;50:1772-86.

4. Descriptors defining levels in the European qualifications framework (EQF). https://ec.europa.eu/ploteus/en/content/descriptors-page (Accessed 1 February 2016).

5. World Health Organisation: world report on ageing and health. 2015 http://apps.who.int/iris/bitstream/10665/186463/1/9789240694811_ eng.pdf?ua=1 (Accessed 16 November 2015).

6. van der Aa MJ, Evers SM, Klosse S, et al. [Reform of long-term care in the Netherlands: solidarity maintained?]. Ned Tijdschr Geneeskd 2014;158:A8253.

7. Dimant J. Roles and responsibilities of attending physicians in skilled nursing facilities. J Am Med Dir Assoc 2003;4:231-43.

8. Koopmans RT, Lavrijsen JC, Zuidema SU. The physician's role in nursing homes: the Dutch solution. Arch Intern Med 2010;170:1406-7.

9. Koopmans RT, Lavrijsen JC, Hoek JF, et al. Dutch elderly care physician: a new generation of nursing home physician specialists. $J$ Am Geriatr Soc 2010;58:1807-9.

10. Ouslander JG, Lamb G, Perloe M, et al. Potentially avoidable hospitalizations of Nursing Home residents: frequency, causes, and costs. J Am Geriatr Soc 2010;58:627-35.

11. Katz PR, Karuza J, Lima J, et al. Nursing home medical staff organization: correlates with quality indicators. J Am Med Dir Assoc 2011;12:655-9.

12. Capaciteitsorgaan. The 2013 recommendations for medical specialist training. Utrecht 2013.

13. Frank C, Seguin R, Haber S, et al. Medical directors of long-term care facilities: preventing another physician shortage? Can Fam Physician 2006;52:752-3.

14. Hauer KE, Durning SJ, Kernan WN, et al. Factors associated with medical students' career choices regarding internal medicine. JAMA 2008;300:1154-64.

15. Petterson SM, Liaw WR, Phillips RL, et al. Projecting US primary care physician workforce needs: 2010-2025. Ann Fam Med 2012;10:503-9.

16. Lovink MH, Persoon A, Koopmans R, et al. Effects of substituting nurse practitioners, physician assistants or nurses for physicians concerning healthcare for the ageing population: a systematic literature review. J Adv Nurs 2017.

17. Yin RK. Case study research design and methods Los Angeles: SAGE, 2014.

18. Pawson R, Tilley N. Realistic evaluation. London: SAGE, 1997.

19. Pawson R, Tilley N. Realist evaluation. 2004 http://www. communitymatters.com.au/RE_chapter.pdf (Accessed 6 January 2015).

20. ter Maten-Speksnijder A, Grypdonck M, Pool A, et al. A literature review of the Dutch debate on the nurse practitioner role: efficiency vs. professional development. Int Nurs Rev 2014;61:44-54.

21. Laurant M, van der Camp K, Boerboom L, et al; Een studie naar functieprofielen, taken en verantwoordelijkheden van physician assistants en Verpleegkundig Specialisten. Scientific Institute for Quality of Healthcare Radboudumc: Nijmegen, 2014.

22. van Vught A, van den Brink G, Harbert K, et al; Physician assistant profession. The Wiley Blackwell Encyclopedia of Health, illness, Behavior, and Society, 2014:1830-2. http://www. platformzorgmasters.nl/publicatie/2013-2/ (Accessed 18 November 2015). 
23. Freund T, Everett C, Griffiths P, et al. Skill mix, roles and remuneration in the primary care workforce: who are the healthcare professionals in the primary care teams across the world? Int J Nurs Stud 2015;52:727-43.

24. Schols JM, Crebolder HF, van Weel C. Nursing home and nursing home physician: the dutch experience. J Am Med Dir Assoc 2004:5:207-12.

25. Bloemendaal I, Albers D, de Kroon S, et al; Taakverschuiving bij de medische zorg vanuit het verpleeghuis. Prismant: Utrecht, 2009.

26. Bakerjian D. Care of nursing home residents by advanced practice nurses. A review of the literature. Res Gerontol Nurs 2008;1:177-85.

27. Abdallah LM. EverCare nurse practitioner practice activities: similarities and differences across five sites. J Am Acad Nurse Pract 2005;17:355-62.

28. Martin-Misener R, Donald F, Wickson-Griffiths A, et al. A mixed methods study of the work patterns of full-time nurse practitioners in nursing homes. J Clin Nurs 2015;24:1327-37.

29. Abdallah L, Fawcett J, Kane R, et al. Development and psychometric testing of the EverCare nurse practitioner role and activity Scale (ENPRAS). J Am Acad Nurse Pract 2005;17:21-6.

30. Wallenburg I, Janssen M, de Bont A. De rol Van de Verpleegkundig Specialist en De Physician Assistant in de zorg. een praktijkonderzoek naar taakherschikking in de tweede- en derdelijnszorg in Nederland. Rotterdam: Rotterdam Instituut Beleid \& Management Gezondheidszorg Erasmus Universiteit Rotterdam, 2015.

31. Flottorp SA, Oxman AD, Krause J, et al. A checklist for identifying determinants of practice: a systematic review and synthesis of frameworks and taxonomies of factors that prevent or enable improvements in healthcare professional practice. Implement Sci 2013;8:35.

32. The nurse practitioner in the Netherlands. http://venvnvs.nl/wpcontent/uploads/sites/164/2015/08/2015-10-30-Factsheet-NursePractitioner-Netherlands-2015.pdf (Accessed 7 April 2016).

33. Timmermans MJ, van Vught $A J$, Wensing $M$, et al. The effectiveness of substitution of hospital ward care from medical doctors to physician assistants: a study protocol. BMC Health Serv Res 2014:14:43.
34. Wat is kwaliteit? http://www.nationaalkompas.nl/preventie/thema-s/ kwaliteit-van-preventie/wat-is-kwaliteit (Accessed 7 May 2015).

35. World Health Organisation: quality of care a process for making strategic choices in health systems. 2006 http://www.who.int/ management/quality/assurance/QualityCare_B.Def.pdf (Accessed 7 May 2015).

36. Liu F, Maitlis S, et al. Nonparticipant observation. In: Mills AJ, Durepos G, Wiebe E, eds. Encyclopedia of Case Study Research. Thousand Oaks: Sage , 2010.

37. Elo S, Kyngäs $\mathrm{H}$. The qualitative content analysis process. J Adv Nurs 2008;62:107-15.

38. Boeije H. Analysis in qualitative research. London: Sage, 2010.

39. Lincoln YS, Guba EG. Naturalistic inquiry. Beverly Hills: Sage, 1985.

40. Sandelowski M. Rigor or rigor mortis: the problem of rigor in qualitative research revisited. ANS Adv Nurs Sci 1993;16:1-8.

41. Punton M, Vogel I, Lloyd R. Reflections from a realist evaluation in progress: scaling ladders and stitching theory. Practice Paper 2016.

42. Abhyankar $P$, Cheyne $H$, Maxwell $M$, et al. A realist evaluation of a normal birth programme. Evidence Based Midwifery 2013;11:112-9.

43. Mason J. Qualitative researching. London: Sage, 2002.

44. Bryant-Lukosius D, Dicenso A. A framework for the introduction and evaluation of advanced practice nursing roles. J Adv Nurs 2004:48:530-40.

45. Schols JM, Crebolder HF, van Weel C. Nursing home and nursing home physician: the dutch experience. J Am Med Dir Assoc 2004;5:207-12.

46. Maier CB. The role of governance in implementing taskshifting from physicians to nurses in advanced roles in Europe, U.S., Canada, New Zealand and Australia. Health Policy 2015;119:1627-35.

47. Maier CB, Aiken LH. Task shifting from physicians to nurses in primary care in 39 countries: a cross-country comparative study. Eur $J$ Public Health 2016;26:927-34.

48. van Vught $A$, van den Brink $G$, Harbert $K$, et al. Physician Assistant Profession. The Wiley Blackwell Encyclopedia of Health, IIIness, Behavior, and Society 2014;2:1830 http://www.platformzorgmasters. $\mathrm{nl} /$ publicatie/2019-2/ 